\title{
LA EVALUACIÓN DE POLÍTICAS \\ PÚBLICAS: UNA CRECIENTE \\ NECESIDAD EN LA UNIÓN EUROPEA.
}

Núm. 1 (2013), pp. 1-30.

Ángel Muñoz ${ }^{1}$, Amelia Pérez Zabaleta², Azahara Muñoz ${ }^{3}$ Cristina Sánchez ${ }^{4}$

Recibido: Febrero, 2013

Aceptado: Marzo, 2013

JEL Clasif: H49, A11, H50, D73

\footnotetext{
${ }^{1}$ Departamento de Economía Aplicada y Estadística UNED (Madrid. España). amunoza@cee.uned.es

2 Departamento de Economía Aplicada UNED (Madrid. España) aperez@cee.uned.es

3 Facultad de Ciencias Empresariales. Universidad Aútonoma de Chile (Santiago. Chile) azahara.munoz.m@gmail.com

4 Departamento de Economía Aplicada y Estadística UNED (Madrid. España). csanchez@cee.uned.es
} 


\title{
Resumen
}

La evaluación de las políticas públicas es un ámbito en expansión de la investigación económica aplicada y de otras múltiples disciplinas como la sociología, la ciencia política y de la administración; afecta especialmente a sectores como la sanidad, la pedagogía o la ingeniería de infraestructuras, y en general todos los que empleen directa o indirectamente recursos públicos; toma especial relevancia tras la creciente preocupación de las autoridades por mejorar la eficacia y la eficiencia del gasto. En el artículo se recoge la experiencia en este ámbito en la Unión Europea y en España. Asimismo, se destacan los principales métodos económicos para la evaluación de las políticas públicas, y se analiza la evolución de la práctica de las mismas en España, derivada principalmente del interés de la Comisión Europea por conocer los resultados de las intervenciones estructurales. El artículo pretende resumir algunas de las motivaciones de los promotores de la nueva Revista de Evaluación de Programas y Políticas Públicas para poner en marcha la misma con objeto de contribuir en el ámbito científico a mejorar la organización de la evaluación en un futuro inmediato.

Palabras clave: Evaluación; Políticas públicas; Burocracia; Eficiencia; Eficacia; Análisis coste beneficio; Análisis coste eficacia; Modelos econométricos.

\begin{abstract}
The evaluation of public policies is an expanding field in applied economy research and in several disciplines such as sociology, political science or management. It especially affects to sectors related to health, education or infrastructural engineering and even more those where the use of public resources become especially important after the growing concern of the authorities to improve their effectiveness and efficiency. This paper describes the experience in the evaluation of public policies in the European Union and Spain. Besides mentioning the main economic methods of evaluation are mentioned. Furthermore, we analyze the evolution of the practices of public policies in the country, derived mainly from the European Commission's interest in knowing the results of structural interventions. This paper aims to summarize the motivations of the promoters of the Journal of Public Evaluation in order to contribute to the discussion and improvements of the practice of evaluation in Spain.
\end{abstract}

Key Words:Evaluation; Public Policy; Bureaucracy; Efficiency; Effectiveness; Cost benefit analysis; cost effectiveness analysis; econometric models. 


\section{Introducción: Los antecedentes en la Evaluación de Políticas Públicas.}

En la actual encrucijada, en la que el sector público se encuentra sometido a una fuerte presión social para aumentar la eficacia y la eficiencia de su gestión, se refuerza la necesidad y utilidad de las evaluaciones de programas y políticas públicas, que debe dejar de ser una cuestión meramente técnica o de cumplimiento de requisitos formales, para pasar a ser un aspecto fundamental de la buena gobernanza; múltiples disciplinas como la economía, la sociología, la ciencia política y de la administración o sectores como la sanidad, la pedagogía o la educación o la ingeniería de infraestructuras, y en general todos los que empleen directa o indirectamente recursos públicos, deben incorporar las técnicas de evaluación a su actividad diaria y convertirla en un elemento fundamental de la misma.

Entendemos que la evaluación de políticas públicas debe formar parte del propio proceso de la "intervención” pública; más allá de su ya obligada regulación legal, los gestores de programas públicos deben evitar que se produzcan desviaciones entre lo previsto y lo realmente ejecutado y valorar el adecuado cumplimiento de los fines y objetivos inicialmente previstos, pronunciándose sobre esta adecuación tanto en términos de eficiencia como de eficacia. Antes de la puesta en marcha de un programa o de una política pública, en la misma fase de diseño y planificación, debe estudiarse, desde la propia necesidad de la misma, su comparabilidad con otras alternativas viables o su mejor estrategia de implementación; durante la ejecución de los programas y por supuesto a la finalización de los mismos es también obligada una reflexión que promueva una mejora de los mismos y su propia continuidad.

La evaluación de las políticas públicas nace en los Estados Unidos, incipientemente en los años 30 del pasado siglo (1933-1937), con la obligatoriedad de evaluar los programas del New Deal de Roosevelt (Ballard, Xavier, 1993), y más tarde como respuesta a la preocupación por valorar los efectos de los múltiples programas sociales emprendidos en la década de los 60 (ayudas para la educación, la salud, la vivienda, etc., enmarcados en la denominada Great Society (Derlien, 1998). Desde entonces ha producido múltiples estudios teóricos y empíricos sobre evaluación de políticas públicas que incorporan las más diversas técnicas de investigación y métodos 
estadísticos propios de las ciencias sociales y que ha convertido a los Estados Unidos, posiblemente, junto a Canadá, en el país con un sistema de evaluación más avanzado.

A principios de los 70 (Jenkins, B., y Grey, A; 1990), en el Reino Unido, se desarrolla un movimiento que reclamaba una mejor gestión en el sector público con la puesta en funcionamiento de sistemas efectivos de control y racionalización del gasto público. Tras el fracaso de sus primeras medidas,- creación del Central Policy Review Stqff (CPRS) y del Program Analysis and Review (PAR) -, la llegada al poder de M. Thatcher en 1979 supuso un nuevo impulso a los estudios sobre el control de los recursos públicos y a la búsqueda de la eficiencia de las administraciones públicas; la creación primero de la Efficiency Strategy (ES) y después de la Financial Management initiative (FMI) implementó un sistema generalizado de estudios y análisis de eficiencia de las administraciones públicas, que, con ciertos cambios, continúa estando operativo.

En esa misma época, la práctica totalidad de los países europeos occidentales (Alemania, Holanda, países nórdicos, Francia, etc.), adoptaron programas socialdemócratas con fuertes políticas de gasto en áreas como la educación, sanidad, vivienda, asuntos sociales etc.; generando la necesidad política de justificar este incremento de gasto y de valorar el impacto posterior de unas intervenciones cuyos efectos reales todavía no habían sido demostrados de forma empírica.

Derlien (1998), constata que a partir de 1969, en la RF de Alemania, la evaluación sería practicada e institucionalizada de forma gradual, tanto a nivel federal como en los estados y ciudades importantes y en el propio Parlamento Federal.

En el mismo sentido, Suecia (Vedung, 1990) puso en marcha un sistema propio de evaluación caracterizado por la incorporación de expertos independientes a múltiples comisiones creadas ad-hoc y por las actuaciones de la Oficina Nacional de Control, Tribunal de Cuentas que, por imperativo legal, desde 1967, se ocupa de evaluar la actividad y la eficiencia con la que actúan las administraciones públicas; parecido modelo es el seguido por la Oficina del Auditor General de Noruega, cuya sección de Auditoría de Rendimientos, tiene como misión la evaluación de la utilización de recursos y bienes públicos en base al análisis crítico de objetivos y resultados (Eriksen, 1990).

En Holanda, en los años 60 y con estos mismos fines, se puso en funcionamiento el COBA (Comité Interdepartamental para el Desarrollo del Análisis de las Políticas); en 
1980 (Bemelmans-Videc et al. 1990) se aprueba la sistematización de la evaluación de las políticas, incorporando la «Sunset Legislation»-con programas que tienen una fecha límite cuya continuación depende de que demuestren su eficacia mediante la correspondiente evaluación de sus efectos- y el Procedimiento de Reconsideración de las Políticas (PRP), que consiste en la inclusión por parte del Consejo de Ministros de un conjunto de programas y servicios, que cada año fiscal deben ser sometidos a PRP, y ser valorados en términos de eficacia y de eficiencia, con el propósito de proponer alternativas que redunden en un menor gasto público, incluyendo la exploración de las posibilidades de privatización o descentralización.

En Francia, a pesar de los intentos llevados a cabo desde los años 70 (Programa de la «Rationalisation des Choix Budgétaires»), la implementación efectiva de los sistemas de evaluación ha sido más tardía (Nioche, 1988); de hecho, hasta 1990 no se estableció la evaluación como procedimiento institucional de revisión de las políticas públicas dentro de la Administración francesa (Decreto 90-82, de 22 de enero de 1990).

Hasta fechas recientes, la práctica de la evaluación ha sido una experiencia atípica aún en España5 (Estivill, 1987; Bustelo, 1998; Zapico, 1998; Alvira, 1993; Pazos, 1996; Fuente, 2002). Sólo para proyectos de gran envergadura se requería la elaboración de análisis coste-beneficio; de hecho, el interés por el análisis y evaluación de las políticas públicas no comienza en España hasta finales de los años 80 con experiencias puntuales, muy alejadas aún de una plena institucionalización de la evaluación (Pazos, 1996) o de la cultura evaluativa (Bachtler y Michie, 1995; Zapico, 1998; Esparcia y Noguera, 2000). Realmente, la evaluación de políticas y programas públicos en España sólo ha mejorado en los últimos años debido a los requerimientos impuestos para el acceso a los fondos comunitarios tras el ingreso en la Unión Europea en 1986.

La reforma de los Fondos Estructurales, en 19886, impuso obligatoriamente, también en España, las evaluaciones sistemáticas y generalizadas, estableciendo que

\footnotetext{
${ }^{5}$ Cansino (2001) señala como precedente de la evaluación, los intentos de implantar en España un presupuesto por programas en la Ley General Presupuestaria de 1977 y en la Ley de Presupuestos Generales del Estado de 1984, si bien, en ambos casos con implicaciones muy limitadas; a pesar de las ventajas, los intentos de ligar evaluación de políticas públicas y procesos presupuestarios han sido complejos y poco frecuentes y enlazados a los intentos de implementación de Presupuesto por Programas.

6 Esa reforma de los Fondos Estructurales aparecía ya en 1986 en el Acta Única, que introdujo en el Tratado una sección V en cuyo artículo 130D se anunciaba la reforma de los fondos orientada a mejorar su eficacia en lo referente a la mejora de la cohesión económica y social (Comisión Europea, 1999b). Previa a la Reforma de 1988 las experiencias son aisladas y fundamentalmente en el marco de las actuaciones del FEDER (Coronado, 1995).
} 
cualquier actuación financiada o cofinanciada por los Fondos Estructurales debía someterse a la obligatoriedad de su evaluación; en este marco, el programa MEANS (Métodos de Evaluación de las Acciones de Naturaleza Estructural)7, cuyo arranque se sitúa en $1994^{8}$, planteó como objetivos propiciar un desarrollo metodológico uniforme y coherente de evaluación a aplicar en toda la UE; los trabajos dieron lugar a una serie de 13 informes acompañados de un glosario de términos para la mejor comprensión.

A partir de entonces, la normativa comunitaria referida al seguimiento y la evaluación ha sufrido cambios importantes dirigidos a dotarla de una mayor precisión, a la implementación de un seguimiento continuado (con evaluación previa, intermedia y final de los programas), a una mayor concreción de los objetivos, al aumento del grado de implicación y de las responsabilidades de los Estados miembros, y a la vinculación de la calidad y los resultados de las evaluaciones con repercusiones financieras para los Estados y las Regiones. Todo ello ha generado un amplio conjunto de directrices y documentos para llevar a cabo el seguimiento y evaluación de programas y proyectos públicos tendente a crear una cultura de la evaluación en las políticas públicas 9 .

\footnotetext{
7 Este programa ha generado múltiples publicaciones, entre las que destacan los seis volúmenes publicados en 1999 (Comisión Europea, 1999). Posteriormente, el programa MEANS se integró dentro de una iniciativa denominada Programa SEM 2000 (Sound and Efficient Management), enmarcada en la línea marcada del artículo 255 del Tratado de Ámsterdam y en la propia Agenda 2000, que pretendía definir una estrategia común entre las autoridades europeas y los Estados miembros en cuestiones relacionadas con la gestión eficiente de los recursos (Cansino: 2001: 1999), donde la evaluación regular de las actividades comunitarias es una parte esencial.
}

8 Durante el periodo de programación correspondiente a los Fondos Estructurales 1989-1993 sólo se requirieron evaluaciones ex post; en el del periodo 1994-1999 se incorporó la exigencia de evaluaciones intermedias; en el periodo 2000-2006, el Marco Comunitario de Apoyo (MAC) se impuso también la evaluación inicial o ex ante. En ese nuevo marco institucional, la evaluación de los programas quedó regulada por el artículo 6(2) del Reglamento (CEE) 2052/88, relativo a la ayuda estructural, y por el artículo 26 del Reglamento (CEE) 4253/88, de coordinación de los fondos, donde se reflejaba la obligatoriedad de evaluar todas las medidas de los Fondos Estructurales.

En 1992, con la firma del Tratado de Maastricht, se incluyó en el artículo 130B que el Consejo debía definir las medidas necesarias para asegurar que los Fondos Estructurales se utilizaban de manera eficaz; por ello en 1993, por el empuje de ciertos Estados miembros, como Holanda y el RU, se refuerza la obligación de evaluar las intervenciones estructurales con la publicación de los Reglamentos (CEE) 2081/93 y 2082/93, que modificaban a los Reglamentos (CEE) 2052/88 y 4253/88 respectivamente

9 Entre los trabajos aplicados en España a consecuencia de estas evaluaciones, destacan los de Salinas (2003), que valora las actuaciones intermedias y finales del FEDER en Castilla y León; Martínez (2003), actuaciones FEDER en las regiones objetivo $n^{0}$ 1; Ogando (2003) para el FSE en Castilla y León; Castillo y Haarich (2003) estudian la intervención en I+D+i en esa misma región; Esteban y Echevarria (1998) analizan la política industrial y tecnológica en distintas regiones europeas; Cadenas y Muñoz (2003) sobre evaluación intermedia del cese anticipado de la agricultura; Tragsatec (2003), para la evaluación intermedia del programa de Forestación de Tierras Agrícolas y del programa agroambiental; Escuela Técnica Superior de Ingenieros Agrónomos U.P.M. y Saborá Sociedad de Estudios (2003), para la medida de indemnización compensatoria en determinadas zonas desfavorecidas, PROINTEC, S.A. para 
En el período de programación 2000-2006 la valoración de la eficacia en la aplicación de los Fondos Estructurales quedaría más reforzada aún, con la experiencia de los informes previos y la obligatoriedad consolidada en los Reglamentos de realizar evaluaciones previas, intermedias y finales para valorar los efectos en relación con los objetivos, no sólo los específicos del programa, sino también los horizontales a todas las políticas comunitarias.

La publicación cada año del Annual Evaluation Review ${ }^{10}$, con una síntesis de los trabajos realizados cada año da un amplio panorama de la situación de la evaluación de políticas en la Unión Europea.

El actual Marco General español para la evaluación de políticas públicas, se establece en el RD 951/2005 de 29 de julio; en 2006 (Ley 28/2006, de 18 de julio), se crea la Agencia Estatal de Evaluación de las Políticas Públicas y de la Calidad de los Servicios (AEVAL); el marco normativo de esta Agencia ha sido posteriormente modificado en varias ocasiones (RD 1039/2009, RD 1083/2009 y RD 256/2012); la Agencia publica anualmente un Informe con el resumen de la situación en España; el borrador de la próxima Ley de Transparencia tiene previsto crear una nueva Agencia de Transparencia, Evaluación de las políticas públicas y calidad de los servicios.

el programa operativo para la mejora de las estructuras y sistemas de producción agrarios en regiones situadas en objetivo $n^{0}$, Quasar (2003), para el Programa Operativo Integrado de Extremadura, etc..

Todos estos trabajos aplicaron mayoritariamente metodologías cuantitativas, con estimaciones estadísticas del impacto de la medida; también son destacables las aportaciones metodológicas de Díez (2001a y 2001b) y de Fuentes (2002), que apuestan por la evaluación participativa, como forma de integrar a distintos agentes en el propio proceso de evaluación y de Viñas (2001), que considera que, por las numerosas dificultades intrínsecas que logra superar y en las que supera a los métodos cuantitativos, la evaluación cualitativa (con entrevistas a la comunidad, ldentrevistas a informadores individuales, grupos de discusión y observación participante), por su menor coste, es la más indicada para la evaluación de las políticas estructurales comunitarias.

Similares informes se llevaron a cabo en todos los Estados Miembros de la UE (Impact assessment of rural development programmes in view of post 2006 rural development policy. DG Agriculture. A report submitted by EPEC. November 2004,

http://ec.europa.eu/agriculture/eval/reports/rdimpact/fullrep_en.pdf

10 Los documentos afectan a múltiples campos con evaluaciones en política agraria, política regional, empleo, asuntos sociales, investigación, sociedad de la información, política de empresas, transporte, energía, política de educación y cultura, política pesquera, sanidad, protección de los consumidores, política ambiental, justicia, fiscalidad, asuntos económicos y financieros, política del mercado interior, política exterior, comercio, Eurostat, servicios de traducción, cooperación, personal y administración, etc..

(http://ec.europa.eu/dgs/secretariat_general/evaluation/documents_en.htm; último publicado para 2009 en http://ec.europa.eu/dgs/secretariat_general/evaluation/docs/final_aer_2009_en.pdf). 
En los últimos años se ha implantado, pues, con alguna, aunque no con la necesaria intensidad, la cultura de la evaluación, con repercusiones organizativas dentro de las instituciones, que han dado lugar a la creación, en algunos casos, de agencias públicas y departamentos especializados responsables de la evaluación en las administraciones, en universidades, y en las empresas, donde hay una nueva línea de negocio dentro de la consultoría para las administraciones públicas y en sociedades y asociaciones de todo tipo especializadas en temas ligados a la evaluación pública.

En España, además de las diversas agencias o institutos públicos (de carácter general como el Instituto Catalán de Evaluación de Políticas Públicas (IVÀLUA), o de carácter sectorial, como las Agencias Regionales de Evaluación de la Calidad Universitaria, las agencias especializadas en el ámbito sanitario etc..). En el ámbito privado, por su parte, en el año 2000 se constituyó en Sevilla la Sociedad Española de Evaluación de Políticas Públicas que tiene como objetivo "contribuir al desarrollo en España de una cultura de la evaluación de políticas públicas como instrumento fundamental de la mejora de la eficacia y la utilidad social de la actividad de las administraciones públicas".

En la misma línea hay que hacer mención a diversas revistas especializadas en España (básicamente la Revista de Gestión y Análisis de Políticas Públicas, editada por Instituto Nacional de Administración Pública y centrada en el ámbito del derecho administrativo y la Revista de la Sociedad Española de Evaluación, editada por la SEE y actualmente en proceso de actualización).

Más abundantes son las publicaciones extranjeras de carácter general (Evaluation Review, Evaluation: The International Journal of Theory, Research and Practice, American Journal of Evaluation, Evaluation and Program Planning, Canadian Journal of Program Evaluation, African Journal of Evaluation) o especializadas (Evaluation \& the Health Professions, Journal of Educational Evaluation for Health Professions, Journal of Evaluation in Clinical Practice, International Journal of Evaluation and Research in Education, etc..

Por todo lo anterior, se deduce el interés en la evaluación de las políticas públicas en los últimos años, en el que profundizamos a continuación con un análisis más detallado de su origen, objetivos, y métodos de evaluación más representativos en el ámbito de la Economía y con especial referencia a la UE. 


\section{Evaluación de políticas públicas: origen, concepto, objetivos y diseño.}

Origen y concepto de la evaluación:

Abordamos en este punto el concepto de evaluación, tomando como punto de partida algunos de los trabajos que constituyen referencias básicas en el este campo. Como reconoce Weiss (1972) se trata de un concepto "elástico" que se emplea para identificar juicios de diferente índole, llegando a constituir un "imán semántico" (Lundquist, 1976). Weiss de hecho no aporta ninguna definición concreta del concepto debido a la diversidad de ámbitos en los que se aplica, pero afirma que lo que tienen todas ellas en común es la valoración de un mérito.

Sriven (1991) por su parte formula una definición generalista del concepto evaluación y considera que evaluación "es el proceso de determinar los méritos, valía e importancia de las cosas" aplicable a cualquier campo de conocimiento, por lo que insiste en el elemento común señalado.

Otra definición interesante es la aportada por Vedüng (1997) que entiende evaluación como la "cuidadosa valoración retrospectiva de los méritos, importancia y valor de la aplicación, productividad y resultados de las intervenciones gubernamentales, que se pretende desempeñe un papel en futuras situaciones y acciones prácticas”.

En esta definición se resalta el carácter retrospectivo de la evaluación, es decir, se trata de una valoración de los logros pasados de una actuación, finalizada o no, lo cual es compatible con las evaluaciones intermedias o finales pero no con las evaluaciones previas a un programa.

Esta posición retrospectiva es complementada por otros autores, como Rossi y Freeman (1989) con aspectos que también incluyen la denominada evaluación previa o evaluación ex ante como instrumento para el diseño de intervenciones y para la toma de decisiones ("la investigación en evaluación es la aplicación sistemática de procedimientos de investigación social para valorar la conceptualización, diseño, aplicación y utilidad de programas de intervención social”). Esta práctica de evaluación ex ante es la adoptada por la propia Comisión Europea para la evaluación de sus 
intervenciones estructurales y coincide con la ampliamente utilizada por la Ciencia Económica con la utilización de métodos como el Análisis Coste-Beneficio.

Un segundo aspecto se refiere al análisis de la productividad de las intervenciones, o lo que es lo mismo, al análisis de eficiencia de la actividad pública.

Finalmente, se resalta un tercer aspecto que entiende la evaluación no como un ejercicio de reflexión carente de proyección hacia el futuro, sino como un esfuerzo orientado a generar un aprendizaje que pueda ser empleado en actuaciones futuras. Se trata de evaluación para la acción, como un componente o una aportación más del proceso de elaboración de políticas públicas, pero realizada sobre intervenciones o programas en ejecución o ejecutados, no sobre programas que no se han puesto aún en marcha.

De todo ello se deduce que la evaluación puede y debe abarcar desde la fase de diseño de las políticas públicas hasta la valoración de resultados una vez ejecutadas, siempre desde una perspectiva crítica que implique la búsqueda de mejoras ante futuras actuaciones e incluyendo todas las esferas del ciclo vital de una política o programa, desde su concepción hasta su impacto pasando por su ejecución.

Una definición bastante aceptada del término evaluación es, pues, aquella que considera que la evaluación consiste en la aplicación de métodos de investigación sistemáticos con el objeto de examinar el diseño, la implementación y la utilidad de las políticas y programas públicos

En el ámbito de la política económica, este esquema es compatible con la definición del término que hacía J. Timbergen "la política económica consiste en la variación deliberada de cierto número de medios para alcanzar determinados objetivos”, asimilando los medios a los inputs y los fines u objetivos a los outputs.

El esquema suele ser más complejo ya que habitualmente los resultados pueden tener diferentes grados de alcance temporal, espacial, sectorial, personal, etc.., por lo que Vedüng (1997) menciona que la evaluación no debe considerar sólo un output, sino que debe hacer referencia a un conjunto de resultados, impactos o consecuencias inmediatas, intermedias y últimas, siendo precisamente la evaluación de las distintas consecuencias más importante que la del simple output de la aplicación de una medida o programa. 
En el ámbito de la evaluación, esas relaciones se denominan de modos distintos, como por ejemplo "modelo" (Weiss, 1972), o "diagrama lógico de impactos" (Comisión Europea, 2000b) o "lógica de intervención" (Isla et al., 1998), si bien lo importante es que se trata de conocer los distintos niveles de resultado a los que da lugar la aplicación de una serie de actuaciones.

Objetivos de la evaluación:

Los motivos para evaluar un programa pueden ser muy diversos, desde los puramente racionales, hasta otros de marcado carácter político (Weiss, 1972).

Los motivos de racionalidad deberían estar orientados a obtener una respuesta objetiva de los resultados logrados por una actuación que a su vez puedan ayudar a decidir sobre el futuro de un programa, como su intensidad, su modo de aplicación, etc. Estas son las funciones de la evaluación que ningún autor discute y que siempre se argumente por quienes la ponen en práctica. De hecho Derlien (1998) destaca tres funciones en la evaluación: la de información, la de asignación y la de legitimación. La primera fue la que predominó durante la fase de despegue de los años sesenta, la segunda durante los años 80 en el proceso de institucionalización y la tercera no es atribuible a un periodo sino que depende del contexto político.

Sin embargo, aparte de estos motivos, puede haber también otros menos legítimos, tales como el deseo de retrasar una decisión, de diluir responsabilidades, de engrandecer o revestir los resultados, de cumplir los requerimientos del sistema de ayudas, de justificar decisiones discutibles, etc.

Como señalaba Monnier (1995), es habitual la "evaluación coartada" o de "lavado de ojos" que selecciona para la evaluación sólo aquellos aspectos que aparentemente son positivos de un programa como forma de justificar el mismo. En la misma línea, otros autores denominan "evaluación de blanqueamiento" a la que trata de ocultar los fallos o "evaluación alegato-prodomo" encargada por la misma organización evaluada para justificar su existencia con respecto a otras instituciones. Además se puede hablar de las "evaluaciones aviso" que recogen indicadores de fracaso para justificar la interrupción de un programa.

A nuestro entender muchas de las evaluaciones llevadas a cabo, tanto en referencia a las acciones estructurales de la Unión Europea, como en otros muchos ámbitos de la 
actividad política en las que se llevan a cabo, tal y como están planteadas actualmente, favorecen el citado "lavado de ojos" y se han convertido en un aspecto burocrático más a solventar; nacido para reforzar las instituciones comunitarias, ha terminado siendo un trámite administrativo que ha dado lugar a la aparición de una Cátedra de “expertos", cuya aportación principal es encarecer el coste de gestión de las políticas y proyectos evaluados.

Ello explica la evaluación positiva que probablemente recibieron en España buena parte de las infraestructuras (aeropuertos, autopistas e instalaciones de todo tipo) construidas en los últimos años y que cualquier análisis riguroso las demuestra claramente inviables, por no mencionar la multitud de programas de intervención pública en cualquier ámbito (social, educativo, sanitario de cooperación internacional, etc.) puestos en marcha en los últimos años.

En este sentido queremos indicar que no siempre una política pública adopta el formato de un programa en el sentido convencional del término; iniciativas políticas como cambios en las leyes impositivas, cambios en la políticas de regulación en sectores más o menos intervenidos, actuaciones de órganos reguladores como la CNMV, Banco de España, CMT, CNE, etc., y deben ser también evaluadas para determinar sus posibles efectos. Baste recordar que en España se ha llevado a cabo todo el proceso de transferencias desde la Administración Central a las CCAA, sin realizar una sola evaluación, ni ex ante, ni ex post de sus consecuencias o resultados.

\section{Diseño de la evaluación:}

Cualquier actuación del sector público debería basarse en un esquema lógico que relacionase los inputs con los outputs determinado el resultado de la evaluación. Sin embargo, como señalan la mayoría de los (Weiss, 1972; Mairate, 2003) lo extraño es encontrar un programa que se articule claramente en fines, objetivos, criterios y bases de decisión.

Al abordar una evaluación de políticas públicas, lo más frecuente, es aplicar la siguiente secuencia: identificar los objetivos, definir la forma de medir la consecución de los mismos a partir de un conjunto de indicadores medibles que concreten los objetivos, las fuentes de información a emplear para obtener información sobre estos indicadores y la forma de llevar a cabo el análisis. 
Aunque pueda parecer sencillo se trata de cuestiones complejas por varios motivos. El primero es que los objetivos de los programas generalmente no son claros, concretos y medibles, sino ambiguos, difusos y difíciles de concretar y medir. Albi (1992) coincide en esa apreciación y además considera que es una de las razones por las cuales en el sector público la eficiencia es más difícil de medir.

En segundo lugar, los programas no solo tienen los objetivos oficialmente declarados, sino que pueden existir otros no declarados en una "agenda oculta", o incluso objetivos inesperados, tanto positivos como negativos.

En tercer lugar, la complejidad se deriva de que los programas públicos suelen incluir una amalgama de actividades, agentes y estructuras, difíciles de identificar. Incluso después de identificados puede ser necesario elegir tan solo un número de ellos clasificados por su importancia relativa, las posibles incompatibilidades, el plazo en el que se manifiestan, etc.

Esta elección lleva implícita la construcción de un modelo con relaciones causa efecto entre las variables (Munger, 2000), que es en definitiva el propio diseño de la evaluación (Weiss, 1972). Después de elegidos los indicadores, podría ser necesario establecer una ponderación para cada uno, así como fijar unos niveles de referencia sobre los niveles deseables de cada objetivo.

La cuarta y última consideración se refiere a una limitación implícita de la utilización de un indicador: puede dar información del resultado de un programa pero no del porqué funciona o no, lo que a veces puede ser interesante conocer.

Identificados los objetivos, los indicadores y el modelo de relaciones entre ellos, el paso siguiente consiste en elegir las fuentes primarias y secundarias de información (datos administrativos de seguimiento, solicitudes y expedientes abiertos, expedientes de gasto, entrevistas con cuestionario abierto y cerrado, etc.)

Finalmente el último paso consistirá en analizar en interpretar los resultados, a partir de la aplicación del modelo o diseño previamente adoptado a lo que se podría añadir el buscar los mecanismos que aseguren la utilidad de la misma. 


\section{Métodos de evaluación en el marco de las Ciencias sociales y de la Economía.}

Independientemente del motivo que impulse la evaluación y de la forma en que se realice, el análisis se centra fundamentalmente en analizar la eficacia de una intervención (Subirats, 1989; Barco, 1996).

En el contexto de la actuación del sector público, la eficacia debe ser entendida como la capacidad de una entidad para lograr los objetivos preestablecidos (Albi, 1992); la eficacia relaciona los outputs reales de una actuación con los inicialmente presupuestados, tratando de valorar el grado de cumplimiento de los objetivos previstos en un periodo de tiempo. En definitiva, se podría definir eficacia como el nivel o porcentaje de objetivos alcanzados con relación a los previstos en un determinado periodo de tiempo.

Sin embargo, para los economistas, junto a la eficacia es necesario hablar de la eficiencia ${ }^{11}$, es decir, la consecución de los objetivos previstos (outputs) con el menor uso de recursos posibles (inputs o costes); dadas las características del sector público, en su evaluación resulta ineludible estudiar la eficiencia de las actuaciones en relación con sus posibles alternativas. Habrá eficiencia si se maximiza la productividad con los recursos que en cada momento se emplean y si el coste de suministro es mínimo. Esta última cuestión se asocia también por parte de algunos autores adscritos a la Ciencia Política con el término de evaluación económica (Guess y Farnham, 2000).

Algunos autores (Müller-Clemm y Burns, 2000) amplían esta visión y piensan que, más allá de la eficiencia y la eficacia, la evaluación de las políticas debe incluir el propio proceso de formulación, ya que parte de su posible éxito exige convencer a mucha gente sobre la validez de las conclusiones (Weiss, 1972) y, en todo caso, tener como fin último, generar efectos positivos futuros en términos de mejora de la toma de decisiones.

Monnier (1993), destaca que la evaluación de la eficiencia es preponderante en los países anglosajones y Weiss (1972) o Antoñanzas (1993), consideran que en todo caso la evaluación debe estar fundamentada científicamente en las Ciencias Sociales, en

\footnotetext{
${ }^{11}$ Monnier (1995) destaca cómo la evaluación de la eficiencia es preponderante en los países anglosajones.
} 
particular en la Ciencia Política y en la Ciencia Económica, con el apoyo de la Sociología, las Ciencias Jurídicas y otras disciplinas como la Estadística.

A pesar de lo indicado anteriormente, en el momento actual existe un importante desacuerdo sobre las bases metodológicas de la evaluación de las políticas públicas ${ }^{12}$ en su conjunto, como señala Vedüng (1997). Por ese motivo, este autor propone una clasificación válida, según sus supuestos, para el conjunto de las Ciencias Sociales, aunque es de especial validez para la Ciencia Política y la Ciencia Económica. El autor distingue entre unos modelos de efectividad y unos modelos económicos. Los modelos de efectividad analizarían los resultados de las intervenciones sin considerar los costes de la intervención, por lo que se trataría de modelos análisis de eficacia. Por el contrario los modelos económicos integrarían la efectividad y el coste, tratándose de modelos de análisis de eficiencia. A estos dos modelos añade un modelo procedimental, más propio de las Ciencias Políticas, que trataría de "verificar la legalidad, equidad, representatividad y otras cualidades de procedimientos, de acuerdo con los cuales se supone que los ministerios, organismos, agencias y otros deben controlar las intervenciones" (Vedüng, 1997), y un modelo profesional de revisión por propios compañeros, conocido también por "peer review” en el ámbito científico.

El modelo procedimental, aunque más próximo a las evaluaciones que se realizan desde el ámbito de la Ciencia Política, tampoco debería descartarse totalmente en las evaluaciones realizadas desde el marco de la Economía pues, muchos economistas hacen caso omiso a las condiciones políticas en las que se formulan e implementan las políticas, considerando que "deben ser el resultado de análisis técnicos reducidos a criterios de eficiencia o equidad. Pero en realidad los fracasos de las políticas públicas generalmente derivan de una incomprensión de la naturaleza de ellas" (Bejarano, 1998). De hecho la consideración del funcionamiento de la burocracia en la gestión de los programas es un aspecto importante de la evaluación porque es "uno de los bienes públicos más importantes es la gestión del Estado: todos resultamos beneficiados cuando la administración es mejor, más eficiente y más sensible” (Stiglitz, 1988).

\footnotetext{
12 Mas (2001), en el contexto de la política estructural de la Unión Europea simplemente afirman que no existe un método superior al resto, sino que depende del contexto en la que tal se realice y generalmente pasa por una combinación de técnicas cuantitativas y cualitativas abierta, en cualquier caso a la experimentación. Turok (1991) mantiene una posición similar respecto a la imposibilidad de fijar un método único de gran aplicabilidad, pues, entre otros las políticas son complejas.
} 
Los Métodos de evaluación más empleados en la Economía son los de análisis de eficacia y los de análisis de eficiencia, sintetizados en ambos casos mediante indicadores.

Los análisis de eficacia pretenden conocer o estimar el output de una determinada intervención pública sin considerar como una variable de estudio el coste de los objetivos al alcanzar; por el contrario, los análisis de eficiencia incluyen este coste y sus posibles alternativas.

En los modelos de análisis de eficacia suelen emplearse como parámetros de evaluación los denominados indicadores ${ }^{13}$.

Los indicadores son magnitudes asociadas a una actividad, a un proceso, a un sistema, etc., que miden cuantitativamente, aunque siempre de forma aproximada, los objetivos a cumplir por la intervención y permiten, por comparación con los estándares, evaluar periódicamente la programación de una medida, el efecto obtenido, el grado de calidad con la que se está ejecutando o ha sido ejecutada, etc.. Los indicadores cumplen, pues, una función descriptiva, aportando información sobre una situación determinada o el estado de un sistema y su evolución en el tiempo, y una función valorativa, que permitir apreciar los efectos provocados por una actuación”.

Su fin principal es ayudar a los actores de la intervención pública a comunicar, negociar o a tomar decisiones de cambio en las formas de gestión, ritmo de los programas, etc.; en el marco de una evaluación, los indicadores más importantes están vinculados al criterio de éxito de las intervenciones.

Algunos autores han establecido las características y o propiedades que deben tener los indicadores. Navarro (1997) ${ }^{14}$, al tratar los indicadores de gestión, como aquellos que permiten el control de la eficacia, eficiencia y economía del gasto, establece que deben cumplir las siguientes propiedades:

\footnotetext{
${ }^{13}$ Debe advertirse, no obstante, que los indicadores son también empleados en los análisis de eficiencia mediante la comparación de inputs aportados y outputs obtenidos (García y Calzado, 1996; Prieto, 2001).

${ }^{14}$ Navarro, A. (1997) "El control económico de la gestión municipal: Un modelo basado en indicadores". Trabajo presentado al II Premi D’investigació Mestre Racional de la Sindicatura de Cuentas de Valencia. Mimeo. Citado por Cansino (2001).
} 
$\checkmark$ Tener la medición de una determinada realidad como objetivo fundamental.

$\checkmark$ Ser la expresión numérica de los objetivos previstos.

$\checkmark$ Permitir realizar una medición no necesariamente monetaria.

$\checkmark$ Facilitar la realización de comparaciones entre distintas entidades o en dos momentos distintos por lo que contribuye a la evaluación.

$\checkmark$ Permitir detectar las acciones que se escapan de los estándares.

$\checkmark$ Permitir valorar la gestión realizada por una determinada entidad.

Otras propiedades que han sido señaladas, Prieto (2001), son las de simplicidad de estimación, precisión, sensibilidad, univocidad, objetividad, pertinencia, accesibilidad, mensurabilidad, fiabilidad, inalterabilidad, imputabilidad, representatividad, adecuación al objetivo y auditabilidad.

En lo que respecta a los análisis de eficiencia, los métodos tradicionalmente más utilizados para evaluar la eficiencia del sector público son el Análisis Coste-Beneficio, el Análisis Coste Eficacia y los análisis econométricos; pueden también emplearse determinados indicadores de eficiencia ${ }^{\mathbf{5}}$.

Antes de profundizar en los métodos consideramos convenientes advertir que, si bien los métodos económicos son imprescindibles, sólo ofrecen una perspectiva parcial y su supuesta superioridad se debe a la fascinación que ofrece su precisión matemática, que lleva a pensar a los responsables de la adopción de programas que ofrecen respuestas definitivas y globales (Vedüng, 1997). Por otra parte destacar que los gobiernos no siempre tienen objetivos de eficiencia y de maximización del bienestar, sino que se pueden plantear las cuestiones en términos de eficacia respecto a objetivos económicos, sociales, políticos y burocráticos (Bejarano, 1998) que los modelos de evaluación económicos no serían capaces de recoger suficientemente.

\footnotetext{
15 Vilardell, 1989; Albi, 1992; Prior et al. 1993; García y Calzado, 1996, Prieto, 2001 y García y Calzado, 1996
} 


\section{Análisis Coste Beneficio:}

El Análisis Coste Beneficio (ACB) se desarrolla dentro la corriente neoclásica y basado en la microeconomía a partir de los principios de la Economía del Bienestar, fundamentalmente en el de compensación de Kaldor-Hicks y en el excedente del consumidor (Munger, 2000; Cansino, 2001) a partir de trabajos de principios del siglo $\mathrm{XX}$. Este principio implica reconocer que existe un beneficio social neto siempre y cuando los que resulten beneficiados con un programa puedan compensar a los perdedores de su pérdida de bienestar y aún así sigan estando mejor.

El ACB, que resulta ser la técnica más completa de valoración de la eficiencia (Albi, 1992), permite tanto decidir entre dos opciones como conocer el beneficio social neto de una determinada actuación en relación a sus costes. Para ello es necesario traducir en términos monetarios la corriente de costes que implica una intervención, así como los beneficios a los que da lugar. Dado que el valor real del dinero no es constante, la comparación actualizada de la corriente de costes y beneficios obliga a emplear una determinada tasa de descuento, generalmente denominada tasa social de descuento (TSD), como más adelante se detalla.

Una vez valorada la corriente de los distintos beneficios y los costes, son varias las reglas de decisión que se pueden adoptar mediante la aplicación de la ACB (Cansino, 1999). La exigencia más común es que la actuación de lugar a un Valor Actual Neto (VAN) mayor que cero, esto es, que en el momento presente los beneficios sociales superen a los costes:

$$
V A N=\sum_{t=0}^{T} \frac{B_{t}-C_{t}}{(1+r)^{t}}>0
$$

Donde $t$ son cada uno de los periodos en los que se manifiestan los costes (C) y beneficios (B) y $r$ es la tasa social de descuento.

Otra regla de decisión del ACB consistiría en exigir que el Ratio Beneficio Coste fuese mayor que 1, o que $i$, la Tasa Interna de Rendimiento (TIR) que iguala beneficios y costes sociales del proyecto, fuese mayor a la tasa social de descuento $r$.

$$
\sum_{t=0}^{T} \frac{B_{t}}{(1+i)^{t}}=\sum_{t=0}^{T} \frac{C_{t}}{(1+i)^{t}}
$$


En caso de poder elegir entre diferentes programas o medidas alternativas el procedimiento llevaría a elegir aquella con mayor VAN, mayor ratio beneficio coste, o mayor TIR. Sin embargo, aunque estos criterios parezcan sencillos presentan en ocasiones problemas que en los trabajos que explican el ACB se recogen extensamente.

\section{Análisis Coste Eficacia:}

El Análisis Coste Eficacia (ACE) comparte con el ACB las mismas bases teóricas, si bien trata de superar las dificultades que surgen resulta difícil medir en términos monerarios un número importante de los beneficios o de los costes sociales (Musgrave y Musgrave, 1994; Cansino, 1999; Mairate, 2003).

El criterio del ACE consiste en comparar los costes sociales de ejecutar una determinada intervención con los resultados de la misma medidos a través de algún índice o indicador no monetario, que permita conocer la efectividad con la que se ha realizado el gasto (Albi, 1992; Cansino, 2001). De otra manera, se puede interpretar también como una comparación de los costes de conseguir el mismo resultado por diferentes procedimientos (Musgrave y Musgrave, 1994).

La comparación se puede realizar de varias formas, por ejemplo observando el coste de un programa aplicado con anterioridad o identificando el coste de un programa similar (Vedüng, 1997). Sin embargo, en muchas ocasiones, es la propia dificultad para encontrar valores de referencia o "benchmarks" para hacer tales comparaciones, lo que dificulta los análisis de eficiencia (Mairate, 2003). La principal limitación frente al ACB es que el método permite indicar cuál es la alternativa más eficiente, pero no asegura que sea socialmente eficiente (Cansino, 1999).

\section{Modelos Econométricos:}

A los anteriores métodos se podrían añadir algunos de los modelos que frecuentemente son empleados en la evaluación de la política regional, tales como modelos input-output, modelos microeconómicos, ${ }^{16}$ modelos macroeconómicos ${ }^{17} \mathrm{O}$ modelos estadísticos, como el análisis factorial o de regresión (Mairate, 2003). Sin embargo, algunos de ellos, como los modelos input-output o los modelos

\footnotetext{
${ }^{16}$ En Magnac (2000) se recoge la aportación de la microeconometría las políticas públicas.

17 La Comisión Europea ha empleado precisamente el modelo econométrico HERMIN para evaluar los efectos de los Marcos Comunitarios de Apoyo en España, Grecia, Portugal, Irlanda y Alemania Oriental (Mairate, 2003).
} 
macroeconómicos, tienen su utilidad condicionada a la dotación de grandes volúmenes de fondos a los programas, puesto que de otra forma no sería posible valorar su efecto sobre las variables económicas dependientes.

Además, en las últimas décadas han comenzado a emplearse para la evaluación del sector público técnicas econométricas de análisis de eficiencia, inicialmente planteadas para la toma de decisiones en la empresa, como forma de superar las limitaciones del ACB y del ACE. Las técnicas se agrupan en dos tipos: por un lado las que toman como referencia de óptimo el comportamiento promedio de las unidades estudiadas y, por otro, las que relacionan la eficiencia de una unidad con el mejor comportamiento mostrado por alguna unidad, siendo este el comportamiento una frontera de eficiencia (Cansino, 1999; cap. 7). Estos planteamientos pueden ser de gran utilidad para la evaluación de programas cuando precisamente haya incertidumbre sobre lo que se considera o no eficiente.

\section{Reflexiones finales.}

Las técnicas para la evaluación de las políticas públicas se emplearon de manera habitual en los países anglosajones desde hace más de cincuenta años, especialmente en Estados Unidos siendo la experiencia de la mayoría de los países europeos mucho más reciente y escasa.

El impulso europeo para la evaluación de las políticas públicas tiene su origen en la obligación impuesta por la Comisión para la evaluación de los programas estructurales $y$, posteriormente de otras iniciativas comunitarias, lo que ha fomentado que en la actualidad las prácticas evaluativas estén implantadas en bastantes ámbitos y en todos los países de la Unión, incluidas las políticas nacionales, en lo que se denomina proceso de institucionalización de la evaluación.

Consideramos que este ejercicio de análisis es positivo para mejorar la eficacia, la eficiencia e incluso la legitimidad del gasto del gasto público, aunque existe el riesgo de que se convierta en un aspecto burocrático más que, simplemente, debe solventarse, primando las evaluaciones justificativas o de coartada.

Es por ello importante definir métodos de evaluación objetivos, mejorando los esfuerzos de la Comisión Europea en este terreno y vigilar e implementar métodos de 
análisis de eficiencia que complementen los estudios de eficacia con los análisis de costes y del estudio de acciones alternativas.

Somos conscientes de la dificultad y de la complejidad de los análisis de eficiencia, pero más allá de las dificultades técnicas, creemos que hay que incorporar elementos que permitan superar las trabas de políticos y burócratas y faciliten que los evaluadores también emitan, en su caso, juicios negativos y severos de las intervenciones públicas evaluadas.

Consideramos de hecho que una de las medidas de la calidad o de la eficiencia de la evaluación de un país o de un conjunto de políticas, debe venir dada por la proporción de evaluaciones negativas y por las supresiones o correcciones efectuadas en los programas por presión o indicación de la evaluación.

Para ello se hace imprescindible la intervención de evaluadores externos e independientes, que aseguren la objetividad de los trabajos, quedando protegidos por algún sistema deontológico, que proteja la relación de presión o clientelismo burócrataevaluador; el modelo de auditoría financiera contable, con ciertas correcciones, podría ser tenido en cuenta para conceder un margen de libertad mayor a los evaluadores; la presencia de universidades y equipos de investigación objetivos también es un aspecto que debe ser tenido en cuenta en este campo.

Finalmente otro elemento a tener en cuenta es la publicidad de las evaluaciones; los últimos destinatarios de las políticas públicas y los contribuyentes que generan los recursos para implementarlas son los ciudadanos; las evaluaciones no deben, pues, tomarse como documentos técnicos para uso interno, los contribuyentes y los ciudadanos deben tener total acceso a ellas, de forma que los firmantes de una hipotética evaluación de coartada o de las denominadas evaluación alegato-prodomo, tenga el adecuado coste personal o profesional si el tiempo demuestra el fracaso absoluto de las previsiones consideradas en las evaluaciones previas, intermedias o incluso finales.

Esta Revista, quiere contribuir, en el ámbito académico y en la medida de sus posibilidades al objetivo principal aquí descrito, en síntesis, mejorar la eficacia y la eficiencia de las políticas y las actuaciones públicas, potenciando su legitimidad y procurando resultados que mejoren las acciones futuras. 


\section{Referencias Bibliográficas}

Albi, E. (1992), "Evaluación de la eficiencia pública. El control de la eficiencia del sector público”, Hacienda Pública Española, nº 120-21, pp. 299-316.

Alvira Martín, F. (1993), "La evaluación en el sector público”, Ekonomiaz, nº 26, pp. 94-103.

Antoñanzas, F. (1993), "La evaluación económica en España: el camino recorrido", Ekonomiaz, $\mathrm{n}^{\circ} 25$, pp. 59-69.

Bachtler, J. y Michie, R. (1995), “A new era in regional policy evaluation?”, Regional Studies, vol. 29 (8), pp. 745-751.

Ballard, Xavier, "Evaluación de Políticas. Marco conceptual y organización institucional”. Revista de Estudios Políticos (Nueva Época). Núm. 8o. Abril-Junio 1993. Pág 199 y siguientes.

Barco, J. del (1996), "El control de eficacia: Significado y contenido del control financiero de programas”, Presupuesto y Gasto Público, nº 18, pp. 173-187.

Bejarano, J.A. (1998), Economía de la Agricultura. Tm Editores, Santa Fé de Bogotá.

Bemelmans-Videc, M. L.; Elte, R.; Koolhaas, E.: (1990) «Policy Evaluation in the Netherlands: Institutional Context and State of Affairs», en R. C. RIST: Program Evaluation and the Management of Government, New Brunswick, Transaction Publishers.

Bustelo, M. (1998), "Deontología de la evaluación: el modelo de los códigos éticos anglosajones”, Gestión y Análisis de Políticas Públicas, nº 11-12 (enero-agosto), pp. 141-156.

Cadenas A. y Muñoz A. (2003) "Evaluación Intermedia de la medida de cese anticipado de la actividad agraria (Programa de Medidas de Acompañamiento 2000-2006. MAPA-UAM. 2003; cit. Impact assessment of rural development programmes in view of post 2006 rural development policy. DG Agriculture. 
Cansino, J.M. (1999): La eficiencia del sector público: Métodos de evaluación y organismo responsables en España. Instituto de Estudios Fiscales, Madrid.

Castillo, J.; y Haarich, S.N. (2003), "La evaluación de las actividades de I+D+i en las regiones objetivo 1: Resultados y perspectivas”, en Ogando, O. y Miranda, B. Evaluación de Programas e Iniciativas Comunitarias: Experiencias, nuevas orientaciones y buenas prácticas. Instituto de Estudios Europeos, Universidad de Valladolid, Valladolid.

Comisión Europea; Legislación y otros documentos de la Unión Europea

- COMISIÓN EUROPEA (1988), Reglamento (CEE) 2054/88 del Consejo, de 24 de junio de 1988, Reglamento Marco de la reforma de los Fondos Estructurales.

- (1994), Reglamento (CE) 1164/1994 del Consejo, de 16 de mayo de 1994, por el que se crea el Fondo de Cohesión, Diario Oficial de las Comunidades Europeas de 25 de mayo.

- (1997), Agenda 2000. Por una Unión más fuerte y más amplia, COM (97) 2000, presentada el 16 de julio.

- (1998), Informe especial núm. 15/98 sobre la evaluación de las intervenciones de los Fondos Estructurales para los períodos 1989-1993 y 1994-1999, acompañado de las respuestas de la Comisión, Diario Oficial de las Comunidades Europeas de 16 de noviembre.

- (1999a), Evaluación de los programas de desarrollo rural 2000-2006 financiados con cargo al Fondo Europeo de Orientación y Garantía Agrícola. Directrices, Dirección General de Agricultura, 48 pp.

- (1999b), Evaluación para una mejor gestión. Revisión intermedia de los programas estructurales. Objetivos núms. 1 y 6 (1994-1999), Oficina de Publicaciones Oficiales de las Comunidades Europeas, Luxemburgo, 60 pp.

- (1999c), Évaluer les programes socio-economiques, Colección MEANS, Luxemburgo, 6 vols. 
- (1999d), Guía para la evaluación del impacto en función del género, Dirección General de Empleo, Relaciones Laborales y Asuntos Sociales, 12 pp.

- COMISIÓN EUROPEA (1999f), Indicadores de seguimiento y evaluación: orientaciones metodológicas, El nuevo período de programación 2000-2006: Documentos de trabajo metodológicos, Documento de Trabajo 3, Dirección General XVI, Política Regional y Cohesión, Coordinación y Evaluación de Operaciones, $67 \mathrm{pp}$.

- (1999g), La evaluación previa de las intervenciones en el período 2000-2006, El nuevo período de programación 2000-2006: Documentos de trabajo metodológicos, Documento de Trabajo 2, Dirección General XVI, Política Regional y Cohesión, Coordinación y Evaluación de Operaciones, 41 pp.

- (1999h), Orientaciones para la evaluación ex-post de los programas del Objetivo 5b. Período de programación 1994-1999, Dirección General de Agricultura, 20 pp.

- (1999i), Reforma de los Fondos Estructurales, 2000-2006. Análisis comparado, $31 \mathrm{pp}$.

- (1999j), Reglamento (CE) 1257/1999 del Consejo, de 17 de mayo de 1999, sobre la ayuda al desarrollo rural a cargo del Fondo Europeo de Orientación y de Garantía Agrícola (FEOGA) y por el que se modifican y derogan determinados reglamentos, publicado en el Diario Oficial de las Comunidades Europeas de 26 de junio.

- (1999k), Reglamento (CE) 1260/1999 del Consejo, de 21 de junio de 1999, por el que se establecen disposiciones generales sobre los Fondos Estructurales, publicado en el Diario Oficial de las Comunidades Europeas de 26 de junio.

- (1999l), Reglamento (CE) 1263/1999 del Consejo, de 21 de junio de 1999, relativo al instrumento financiero de orientación de la pesca, publicado en el Diario Oficial de las Comunidades Europeas de 26 de junio. 
- (1999m), Reglamento (CE) 1264/1999 del Consejo, de 21 de junio de 1999, que modifica el Reglamento (CE) 1164/1994 por el que se crea el Fondo de Cohesión, publicado en el Diario Oficial de las Comunidades Europeas de 26 de junio.

- (1999n), Reglamento (CE) 1265/1999 del Consejo, de 21 de junio de 1999, que modifica el Anexo II del Reglamento (CE) 1164/1994 por el que se crea el Fondo de Cohesión, publicado en el Diario Oficial de las Comunidades Europeas de 26 de junio.

- (1999p), Reglamento (CE) 1783/1999 del Parlamento Europeo y del Consejo, de 12 de julio de 1999, relativo al Fondo Europeo de Desarrollo Regional, publicado en el Diario Oficial de las Comunidades Europeas de 13 de agosto.

- (1999q), Reglamento (CE) 1784/1999 del Parlamento Europeo y del Consejo, de 12 de julio de 1999, relativo al Fondo Social Europeo, publicado en el Diario Oficial de las Comunidades Europeas de 13 de agosto.

- (1999r), Sexto informe periódico sobre la situación y la evolución socioeconómicas de las regiones de la Unión Europea, Luxemburgo, 242 pp.

- COMISIÓN EUROPEA (200of ), Las acciones estructurales 2000-2006. Comentarios y reglamentos, Oficina de Publicaciones Oficiales de las Comunidades Europeas, Luxemburgo, 158 pp.

- UNIÓN EUROPEA (1992), Tratado de la Unión Europea, firmado en Maastricht el 7 de febrero de 1992.

- (1995), «Mesurer les efects sur l'emploi des interventions structurells communautaires»,

- Cuadernos MEANS, núm. 3, Bruselas, 63 pp. 
- (2000a), Communication from the Commission to the Member States of 28.4.00 laying down guidelines for a Community Initiative concerning economic and social regeneration of cities and of neighbourhoods in crisis in order to promote sustainable urban development. URBAN II, C(2000) 1100, 15 pp.

- (200ob), Comunicación de la Comisión a los Estados miembros de 14.4.00 por la que se establecen las orientaciones relativas a los Programas de Iniciativas Comunitarias para los que se invita a los Estados miembros a presentar propuestas para la concesión de subvenciones en el marco de la iniciativa EQUAL, C(2000) 1101, 38 pp.

- (2000c), Comunicación de la Comisión a los Estados miembros de 14.4.00 por la que se fijan las orientaciones sobre la Iniciativa Comunitaria de desarrollo rural (LEADER+), C(2000) 946, 15 pp.

- (2000d), Comunicación de la Comisión a los Estados miembros de 28.4.00 por la que se fijan las orientaciones para una iniciativa comunitaria relativa a la cooperación transeuropea para fomentar un desarrollo armonioso y equilibrado del territorio europeo. INTERREG III, C(2000). 1101, 38 pp.

- (2000e), Evaluation ex-ante et indicateurs pour INTERREG, El nuevo período de programación 2000-2006: Documentos de trabajo metodológicos, Documento de Trabajo 6, Dirección General XVI, Política Regional y Cohesión, Coordinación y Evaluación de Operaciones, $31+6$ pp.

Derlien Hans-Ulrich (1998) "Una comparación internacional en la evaluación de las políticas públicas. Gestión y análisis de políticas públicas”, No 11-12, 1998, págs. 13-22.

Ericksen, B.: «Institucional Aspects of Evaluation in Norway», en R. C. RIST: Program Evaluation and the Management of Government, New Brunswick, Transaction Publishers, 1990. p. 127

Esparcia, J.; y Noguera, J. (2000), “Aproximación técnico-metodológica de la cultura evaluativa y la evaluación de programas de desarrollo rural", Cuadernos de Geografía (Universidad de Valencia), nº 67, pp. 77-101. Universidad de Valencia. 
Esteban, M.; y Echebarria, C. (1998), Metodologías y Procedimientos de Evaluación. El caso de la Política Industrial y Tecnológica. Servicio Editorial, Universidad del País Vasco, Bilbao.

Ericksen, B.: «Institucional Aspects of Evaluation in Norway», en R. C. RIST: Program Evaluation and the Management of Government, New Brunswick, Transaction Publishers, 1990.

Estivill, J. (1987), “Notas sobre la evaluación en España”, Revista de Trabajo Social, n ${ }^{0}$ 6, pp. 91-97.

Fuentes, F. (2002), "Evaluación de políticas públicas: Evaluación participativa y situación en España”, Análisis Local, nº 2002-4, pp. 43-49.

García Sánchez, M.I. (2000) "El papel de los indicadores de perfomance en la evaluación de políticas públicas”, Actualidad Financiera, $n^{0} 5$ (4), pp. 75-82.

Guess, G.M.; y Farnham, P.G. (2000), Cases in public policy analysis, $2^{\mathrm{a}}$ Ed. Georgetown University Press, Washington D.C.

Jenkins, B., Y Grey, A.: «Policy Evaluation in British Government: From Idealism to Realism», en R. C. RIST: Program Evaluation and the Management of Government, New Brunswick, Transaction Publishers, 1990.

Isla, M.; Mella, J.M.; y Soy, A. (1998), “La política regional de la UE y su evaluación”, en Mella, J.M. Economía y política regional en España ante la Europa del siglo XXI. Akal Textos, Madrid.

Lundquist, L. (1976) "Algunos puntos de vista sobre el concepto de planificación positiva”. Statsventenkaplig Tidskrift, 121-139.

Magnac, T. (2000), "L'apport de la microéconométrie à l'evaluation des politiques publiques", Cahiers d'economie et sociologie rurales, $n^{0}$ 54. pp. 89-113. 
Mairate, A. (2003), "La evaluación de los fondos estructurales: Aspectos metodológicos y teóricos”, en Ogando, O.; y Miranda, B. Evaluación de Programas e Iniciativas Comunitarias: Experiencias, nuevas orientaciones y buenas prácticas. Instituto de Estudios Europeos, Universidad de Valladolid, Valladolid.

Mas, M. (2001), "La evaluación de políticas regionales: Lecciones de intervenciones comunitarias”, Revista Valenciana de Economía y Hacienda, nº 1, pp. 83-95.

Monnier, E. (1993), “Objetivos y destinatarios de las evaluaciones”, Documentación Administrativa, $\mathrm{n}^{0}$ 224-225, pp. 131-152.

Müller-Clemm, W.J.; y Burns, J.A. (2000), "Evaluación del proceso: hacia un asesoramiento de políticas fundamentado en pruebas”, Presupuesto y Gasto Público, nº 24, pp. 33-50.

Munger, M.C. (2000), Analyzing Policy. Choices Conflicts and Practices. W.W. Norton, New York.

Musgrave, R. A. y Musgrave, P.B. (1994), Hacienda pública teórica y aplicada ( 5 a Ed). Mc Graw-Hill, Madrid.

Navarro, A. (1997) "El control económico de la gestión municipal: Un modelo basado en indicadores”. Trabajo presentado al II Premi D’investigació Mestre Racional de la Sindicatura de Cuentas de Valencia. Mimeo.

Nioche, J. P. (1988): «Accountable Public Management», En A. Kakabadse, P. Rondo. Broveto Y R. Holzer (eds.): Management Development and the Public Sector, Avebury, Gower Publishers, 1988.

Ogando Canabal, O. (2003), "La evaluación intermedia del programa operativo regional FSE de Castilla y León (1994-1999)”, en Ogando, O.; y Miranda, B. Evaluación de Programas e Iniciativas Comunitarias: Experiencias, nuevas orientaciones y buenas prácticas. Instituto de Estudios Europeos, Universidad de Valladolid, Valladolid. 
Pazos, M. (1996), "La evaluación de programas en la administración pública española: el estado de la cuestión”, Papeles de Trabajo, $n^{0}$ 16/96, Instituto de Estudios Fiscales, Madrid.

Prieto Jano, M.J. (2001), "Los indicadores de gestión como instrumento de medición de las actuaciones del sector público”, Presupuesto y Gasto Público, nº 26, pp. 83110 .

Prior, D.; Verges, J.; y Vilardell, I. (1993), La evaluación de la eficiencia en los sectores privado y público. Instituto de Estudios Fiscales, Ministerio de Economía y Hacienda, Madrid.

Rossi, P.H.; y Freeman, H. (1989), Evaluation: A systematic approach. Sage, California.

Salinas Sánchez, J. (2003), "La evaluación intermedia del programa operativo regional FEDER de Castilla y León (1994-1999)”, en Ogando, O., y Miranda, B. Evaluación de Programas e Iniciativas Comunitarias: Experiencias, nuevas orientaciones y buenas prácticas. Instituto de Estudios Europeos, Universidad de Valladolid, Valladolid.

Stiglitz, J.E. (1988), La economía del sector público, (2 ${ }^{\mathrm{a}}$ ed.). Antoni Bosch Editor, Barcelona.

Subirats, J. (1989), Análisis de políticas públicas y eficacia de la Administración. Ministerio para las Administraciones Públicas, Madrid.

Timbergen, J. (1952) On the Theory of Economic Policy.

Turok, I. (1991), "Policy Evaluation as a Science: A Critical Assessment”, Applied Economics, vol. 23 (9) pp. 1543-1550

Vedüng, E. (1990). «Development of Evaluation in the Swedish Political System». Conferencia Internacional de La Haya sobre Evaluación de Políticas y Programas.

Vedüng, E. (1993), "Utilización de la evaluación”, Revista de Servicios Sociales y Política Social, no 30 , pp. 69-80. 
Vilardell Riera, I. (1989), "La evaluación de la eficiencia de las actividades de la Administración pública”, Economía Pública, nº2, p. 47-60.

Viñas, V.E. (2001), "El uso de técnicas cualitativas en la evaluación de programas. Los programas de desarrollo regional financiados por la Unión Europea”, Revista Española de Investigaciones Sociológicas, nº 95, pp. 155-177.

Weiss, C.H. (1972), Evaluation Research: Methods of Assessing Program Effectiveness. Prentice Hall, New Jersey.

Zapico Goñi, E. (1998), "La integración de la evaluación de políticas públicas en el proceso presupuestario”, Gestión y Análisis de Políticas Públicas, no 14-15 (eneroagosto), pp. 53-59. 